\title{
Aurama: caregiver awareness for living independently with an augmented picture frame display
}

\author{
Pavan Dadlani · Alexander Sinitsyn • \\ Willem Fontijn · Panos Markopoulos
}

Received: 24 April 2009/ Accepted: 23 November 2009/Published online: 6 January 2010

(C) The Author(s) 2010. This article is published with open access at Springerlink.com

\begin{abstract}
Aurama is a system designed to provide peace of mind and a sense of connectedness to adults who care for elderly parents living alone. Aurama monitors the elders at home using unobtrusive sensor technology and collects data about sleeping patterns, weight trends, cognitive abilities and presence at home. The system provides an unobtrusive ambient information display that presents the status of the elder and lets its users inspect long-term data about the well-being of the elder interactively. Aurama was designed iteratively with substantial user involvement through interviews, prototype evaluation, focus groups and lab tests. The final prototype was evaluated in two field trials each involving an elder and their adult children. The input of users throughout the design process and during these tests demonstrates clearly the potential of awareness systems to support the target user group to obtain peace of mind and feel connected. Furthermore, the users indicate a clear need for information on long-term trends relating to the well-being of aging parents, in contrast to the current emphasis in this field of research on
\end{abstract}

P. Dadlani $(\varangle) \cdot$ A. Sinitsyn

Philips Research, Eindhoven, The Netherlands

e-mail: pavan.dadlani@philips.com

A. Sinitsyn

e-mail: alexander.sinitsyn@philips.com

W. Fontijn

Serious Toys, Eindhoven, The Netherlands

e-mail: willem.fontijn@serioustoys.com

P. Markopoulos

Department of Industrial Design,

Eindhoven University of Technology,

Eindhoven, The Netherlands

e-mail: p.markopoulos@tue.nl providing instantaneous status information about daily activities and context.

Keywords Awareness systems - Social connectedness · Ambient assisted living $\cdot$ Health monitoring

\section{Introduction}

The aging of the population in developed countries is a well-established fact. Extrapolating current trends, $53 \%$ of the total population of Europe will be above 65 by $2050^{1}$. This trend inflates the 'sandwich' generation: adults with children and an aging parent to take care of. The members of this group can be classified into drastic life changers, significant contributors and peripherally involved (Consolvo et al. 2004). Drastic life changers make major changes to their own life to care for the elder (e.g., sacrificing a career, hobbies or even their own family life). Significant contributors provide regular care for the elder; this has a major impact on their own life, but they are still able to maintain their own independent lifestyle. The peripherally involved typically live at a distance from the elder and provide care that is meaningful, but typically irregular and the type of help they provide is most commonly social (e.g., visits and phone calls) and house maintenance. Providing this support is hampered by distance and the difference in pace of life.

Given the current trends of an aging society and its socio-economic impact, it is highly desirable to be able to support the elderly to extend their stay in their own home as long as possible, while maintaining and enhancing their

\footnotetext{
${ }^{1} \mathrm{http} / / / \mathrm{epp}$. eurostat.ec.europa.eu/tgm/table.do?tab=table \&init=1\& language $=$ en $\&$ pcode $=$ tsdde $511 \&$ plugin $=1$
} 
autonomy and quality of life. This can be achieved by improving the support received from the elder's own environment in which the use of Ambient Intelligence (AmI) can play an important role. AmI refers to electronic systems that are sensitive and responsive to the presence of people (Aarts et al. 2001). It is a paradigm envisioned to materialize in the next few years (Aarts and Marzano 2003), when the home is expected to become populated by large number of 'smart' devices interconnected via an invisible web of networked services (Aarts et al. 2001).

The class of systems we concern ourselves with is often referred to as 'awareness systems' to indicate that their primary purpose is to create awareness of context rather than be goal-oriented or provide efficient information exchange. Several researchers worldwide have explored ways in which awareness systems can support independent living. We report research that explores the potential of such systems to offer peace of mind to adults that are peripherally involved in the care for their elderly parent(s) and to bring about that both sides feel interconnected. More specifically, we report on the use of ambient intelligence technology to let adult children be aware of the well-being of a remote elder. This general scenario has been shown to hold promise by earlier research such as the CareNet display (Consolvo et al. 2004), the Digital Family Portrait (DFP) (Mynatt et al. 2001; Rowan and Mynatt 2005) and the Diarist system (Metaxas et al. 2007). These earlier research prototypes are discussed in the next paragraph.

The CareNet project (Consolvo et al. 2004) investigated visualizations of information that is useful to individuals who are responsible for the daily care of an elder, such as drastic life changers and significant contributors. It involves information on medication, outings, meals, activities, mood, falls and calendar items. The visualizations were evaluated during a 3-week trial with four pairs of elderly and their children. It was concluded that the quality of care can improve through using such a system. The information collection was simulated [Wizard of Oz method (Kelley 1984)], leaving questions open regarding the design and potential acceptance of the sensor infrastructure.

The DFP (Mynatt et al. 2001; Rowan and Mynatt 2005) emphasized the need to tie awareness displays to everyday objects, like a photo frame. A sensor infrastructure provides information regarding the general level of activity of the elder and this can be inspected interactively by the child. The prototype was deployed at the home of an elder living alone and her son. Their experience of using the system was studied over a period of 6 weeks. This illustrated the benefits of awareness systems for fighting feelings of loneliness of the elder and for offering peace of mind to the child.

The Diarist system (Metaxas et al. 2007) follows a similar approach to the DFP but provides a semantically higher level of interpretation of the sensed activities. It detects the activities of daily life of the elder and informs their child remotely. The selection of the everyday activities that are monitored and displayed was arrived at iteratively through user surveys, interviews and focus group studies that established the preferences of both children and their parents. As reasoning about humans and their activities is inherently complex on its own, the design and the evaluation focused on how to present such semantic interpretations to users. Diarist provided awareness information at several levels: rough presence information that can be seen from a distance, more detailed logs of the daily activities that need to be observed close up and very detailed (synthesized) narratives of activities. A 2-week trial with two pairs of elderly and their peripherally involved children demonstrated its potential for providing peace of mind and connectedness. It demonstrated also the value of a seamful design (Chalmers and Galani 2004) for letting users inspect the inferences that systems make based on user activity and make the users aware of potential flaws of these inferences.

Next to the mentioned research prototypes, there are several products and services commercially available that allow care providers and family members to be informed about the status of an elderly. For example, the HomMed system (Honeywell HomMed http://www.hommed.com/) allows doctors to monitor health parameters of lone elderly patients remotely. It requires, however, the use of obtrusive devices to measure vital signs (weight, heart rate, etc.). Although, family members are not directly involved, such systems provide 'peace of mind' indirectly by knowing that the elder's health is monitored. A system in which family members are involved is the i-POT (Zojirushi Corporation: http://www.zojirushi.com/). The i-POT works as an ordinary hot water dispenser, with the additional feature of being able to track the daily consumption of hot water and inform caregivers about it. The latter can then assess if there are any abnormalities.

Two major initiatives regarding the unobtrusive monitoring of activities of daily living are QuietCare (http://www. quietcaresystems.com/) and HealthSense (http://www. healthsense.com/). The QuietCare's system consists of three components: motion's sensors, a base station and a web-based client where caregivers can get detailed information about the elder's well-being. The system relies solely on motion sensors that are placed around the home and generate information on activities of daily living (ADLs). The ADLs include cooking, interacting with medication, bathroom usage, waking-up and the overall level of activity. The ADL data are sent to their centralized server via the base station. The data are used to assess whether any alerts or notifications should be shown on the website or sent via email, SMS or even by telephone, if the alert is of high severity. HealthSense uses the eNeighbor Resident 
Monitoring system that generates alerts related to significant changes in ADLs, such as significant changes in patterns of sleeping, eating or toilet use. These early indicators can trigger an early intervention by caregivers.

These earlier investigations and commercial products provide converging evidence regarding the potential of awareness systems and offer some ideas on how awareness information could be presented. However, the empirical evidence collected is still limited due to the number of users involved, and the level of realism of the trials. There are still open questions regarding the type of information that needs to be captured and communicated, regarding if and how users would utilize such technologies in realistic circumstances, and regarding concerns users may have about using them. Actual deployments are still very scarce and small scale: for the scenario of remote awareness of a lone elderly there are about 5 actual deployments reported to date (Consolvo et al. 2004; Mynatt et al. 2001; Rowan and Mynatt 2005; Metaxas et al. 2007; Morris et al. 2003) and each explored awareness of different aspects of the elder's life. Hence, the evidence regarding the benefits they provide is still only preliminary and requires elaboration and corroboration.

In the cited work, researchers assumed they needed to monitor a specific type of information to compensate for the distance between the elder and their children, with presence and general activity level as typical candidates to consider. What the actual awareness information needs are (for both parties) were not explored systematically. Also more emphasis can be placed on the esthetic quality of the interaction with an awareness display that is possible and even necessary when considering different hardware platforms than a PC. Arguably, and as our initial user studies showed (see the following sections) this is an important aspect for motivating people to adopt the system, as well as, for supporting the interaction scenarios envisaged.

Morris et al. (2003) carried out ethnographic studies with elderly with dementia and their caregivers. A set of themes of needs and design principles were provided, together with a set of concepts addressing these needs. Social connectedness is one of the themes. The prototype they developed (Morris 2005) includes a social network feedback display, which is continuously updated with self-reported and sensor data, lamps that provide presence information indicating availability of others and contextual cues to trigger and support phone based communication. Morris (2005) implemented their prototype and conducted a three-month in-home field study with six pairs of elderly people and their primary caregivers. Interestingly, the prototypes succeeded not only in providing awareness to the caregiver but encouraged selfreflection and awareness with both parties. Their prototype also included a sensor triggered 'presence lamp' to signal presence to the caregiver and vice versa.

Our investigation contributes to this line of inquiry through the design, deployment and evaluation of Aurama (see Fig. 1). Aurama is an electronic picture frame, adorned with a colored light aura that provides updates of well-being information, and as such part of an end-to-end awareness system. It has some similarities with the related work described above, but as discussed below, it focuses on the presentation of long-term trends or unusual occurrences, rather than just instantaneous awareness information. First, we describe the empirical basis of the design of this system, then, the implementation and deployment in lab and field trials and finally the lessons learnt from the trials.

\section{Preliminary user studies}

To evaluate the concept of providing awareness of an elderly parent's well-being to caregivers and to find out what information they would want to receive in this regard, four focus groups were conducted in the Netherlands (Europe) with peripherally involved caregivers $(N=19$, average age: 49). The discussion covered a list of 50 items in six areas of well-being: health, activities, outings, entertainment, social contacts and security. The list addressed all areas of well-being identified by Mynatt et al. (2001) and was extended to cover more detailed aspects of each category. The findings were further elaborated and
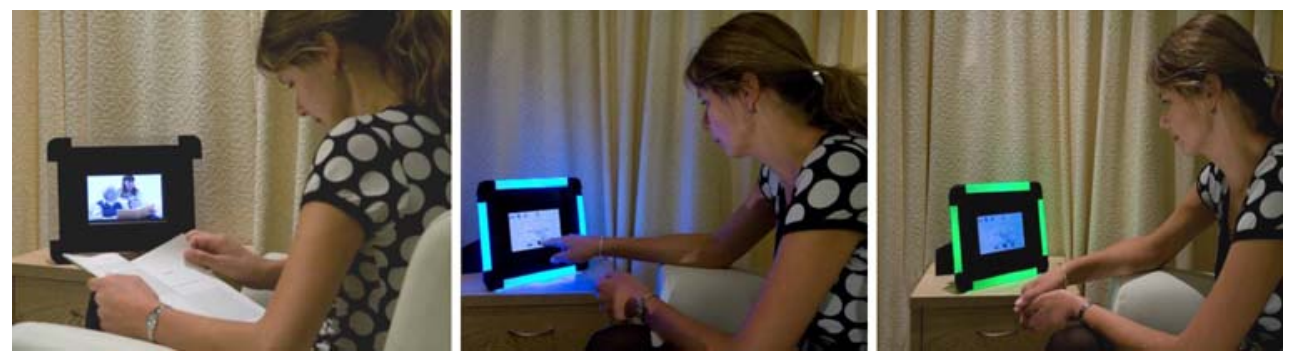

Fig. 1 Aurama: an aware picture frame emitting colored light auras indicating elderly is not at home (left), elderly is at home (center) and there is an abnormal change in the elder's patterns (right) 
verified by one-on-one interviews with caregivers $(N=8$, average age: 44) and with retired seniors $(N=7$, average age: 79).

Based on participants' statements, the phone is the most common means of communication between these caregivers and seniors, followed by visits. When discussing problems in communication, specifically about well-being, children claim that "nothing can replace visits" and most of them hope to pick up on changes when they visit. However, they also claim that "visits do not last long enough to get a clear picture of their well-being". Rather than detailed trivia of daily activities, care giving children want to receive high-level information about their parents' well-being, but only if something is 'not ok' or has changed in their parents' routine. Interestingly, they mentioned that they wish to be informed about gradual changes in lifestyle that may not be easy to notice during visits. Regarding detailed information, they do not want to be confronted with it constantly, but would like to be able to access it upon request to understand unusual situations. They expect to feel obliged to react to unusual events but do not anticipate this to be a burden.

Although they value the possibility to see historical data, they dislike the idea of receiving a daily status report or a 'story' with detailed schedules and activities, as they feel that this would be privacy invasive for their parents. Persistent changes over time in eating habits, sleep patterns and mood are important to them, e.g., when the parent does not eat well for a week. Children want to have access to some level of detail on request in the following situations: (1) whenever they are informed that something may have changed in their parent's routine, (2) whenever they have some worries about their parents and (3) if they just want to be reassured that everything is fine and that the system is still working. Figure 2 shows the average questionnaire responses from the participants concerning the level of interest in detailed information about their parents based on different items in the six categories of well-being.

Caregivers prefer their parents to be reminded directly regarding issues like medication intake and security (e.g., doors open), instead of informing them. They recognize that disease related data and vital signs (under health status) are important to monitor but they would rather have these sent to a doctor who would provide them with richer information and explanations. They do not consider entertainment and social contacts as important to know about. These are considered to be a private matter of their parent. However, they are concerned that their parents' cognitive abilities may decline over time and want to know about it from the onset; even potentially at the cost of invading privacy: "although some things may be private, it is important to know to protect them". Moment to moment information about presence at home is important to children. This can be valuable if they know their parents are at home but do not pick up the phone, suggesting that something could be wrong. This aligns with results in other applications of awareness systems, e.g., for families with young children (Brown et al. 2007) and (Khan and Markopoulos 2009) where a major use of awareness systems was found to be simply for reassurance that everything is as usual.

Several ways of presenting awareness information were discussed including: a digital book, a digital painting, a digital photo frame, photonic textiles, a little robot, a lamp, a mobile phone, an art sculpture, the television and a website. These were presented in a slide show presentation. Among these various possibilities, participants favored interactive digital photo frames, several times referring to quotes such as "we already dedicate photo frames to people we care about" and the fact that photo frames already add a 'personal touch'. Many participants commented that they have photo frames to think about their
Fig. 2 Average responses of perceived interest for receiving detailed information based on various items under each category of well-being information

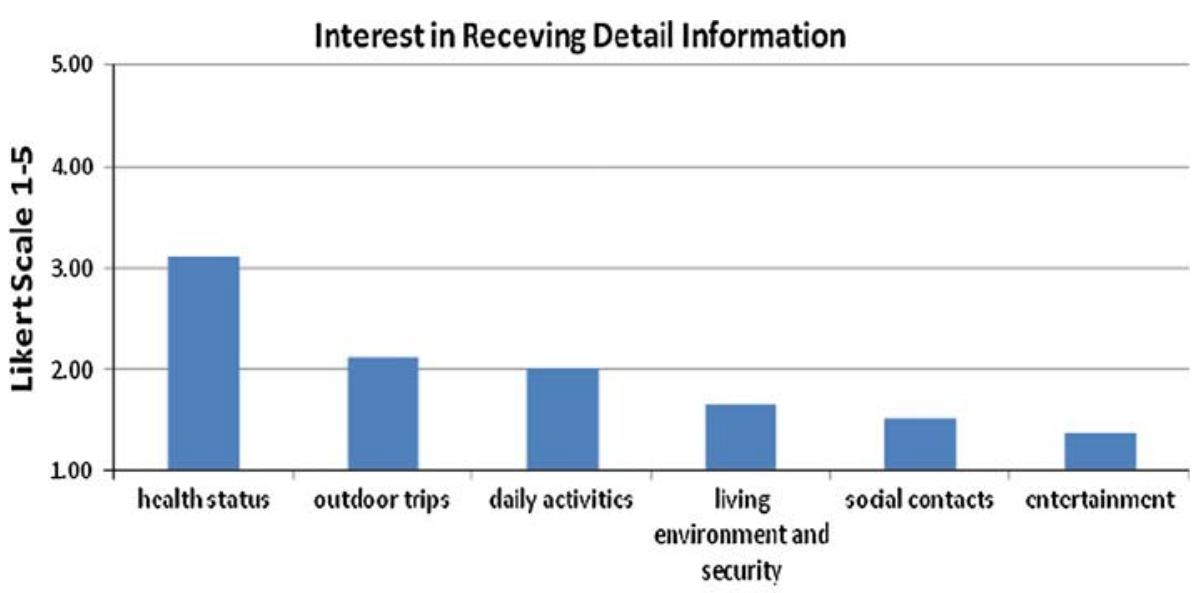

Type of information 
loved ones and feel connected. Participants favored the concept because they are already familiar with photo frames and these would not be a new dedicated gadget, thus, making it easier to blend them into the home environment.

Overall, elderly do not mind sharing information with their children but they fear becoming a burden to them: "they are too busy, they have their own children and their own lives". Most of the elder informants consider themselves 'too young' for our system. Nevertheless, they like it when their children show interest.

Regarding some of the concepts discussed by Morris et al. (2003) on using cameras to monitor elderly and the frail, our initial user studies reveal that elderly would not mind having such systems as long as there are no cameras installed in their home and the system is unobtrusive. This is a challenge that needs to be addressed during the design and implementation of such a system.

\section{The Aurama awareness system}

Our preliminary study showed that contrary to the related systems discussed in the introduction section, there is an interest for longer term monitoring information. We decided to verify this by designing, implementing and evaluating a system presenting information about presence at home, sleep patterns, weight variations, and cognitive ability. Although related work has been done on measuring sleep (Adami et al. 2003) and cognitive decline (Morris et al. 2003), very little has been done to study the effect of tracking and sharing patterns of these with caregivers.

\subsection{System architecture}

Figure 3 shows the system's architecture. The core of the architecture is a database management system that stores persistently and manages all data collected from the various sensors and algorithms of the system in a structured way. The database allows for performing sensor data analysis over a long period of time. It also is the interface between different components of the system (e.g., sensors, algorithms, clients). The data analyzed is exposed via a web server running on a PC in the elder's house.

The choice of sensors to be used in the system was dictated by the following requirements: reliable measurements of observable events insuring correct conclusions based on sensed data, ease of installation, unobtrusiveness and the sensor battery life should be sufficient to run a continuous field trial without intervention.

A wireless tag from Sensite Solutions (http://www. sensite-solutions.com/), as shown in Fig. 4 (left), transmitting radio-frequency (RF) signals and placed on a keychain was used to detect presence at home. The presence status is monitored constantly, updating information on the server. A client process at the child's home polls the server for an updated status every minute. Our initial tests have shown that the presence detection based on RF signal strength alone generates a high number of false alarms, e.g., the user could be reported to be 'out of home' while in fact the user was at home. Furthermore, the RF-based presence detector is susceptible to false readings within a building due to the irregular RF field patterns generated within a building. We have overcome this problem by adding an accelerometer to the RF signal transmitter. By fusing data from both RF signal strength detector and accelerometer, we could obtain more reliable presence detection. Information fusion (i.e., the combination of multiple information sources) is commonly used in different application domains to obtain improved information for detection and classification (Nakamura et al. 2007). In our case, adding the redundancy (i.e., using 2 complementary sensors instead of one) makes the presence detection less vulnerable to failure of a single sensor.

Weight is measured with load sensors from Vetek (http://www.vetek.com/) (Fig. 4 right) placed under each leg of the elder's bed using a similar technique as (Adami et al. 2003). A measurement is taken daily during the night.

Fig. 3 An overview of the system's architecture

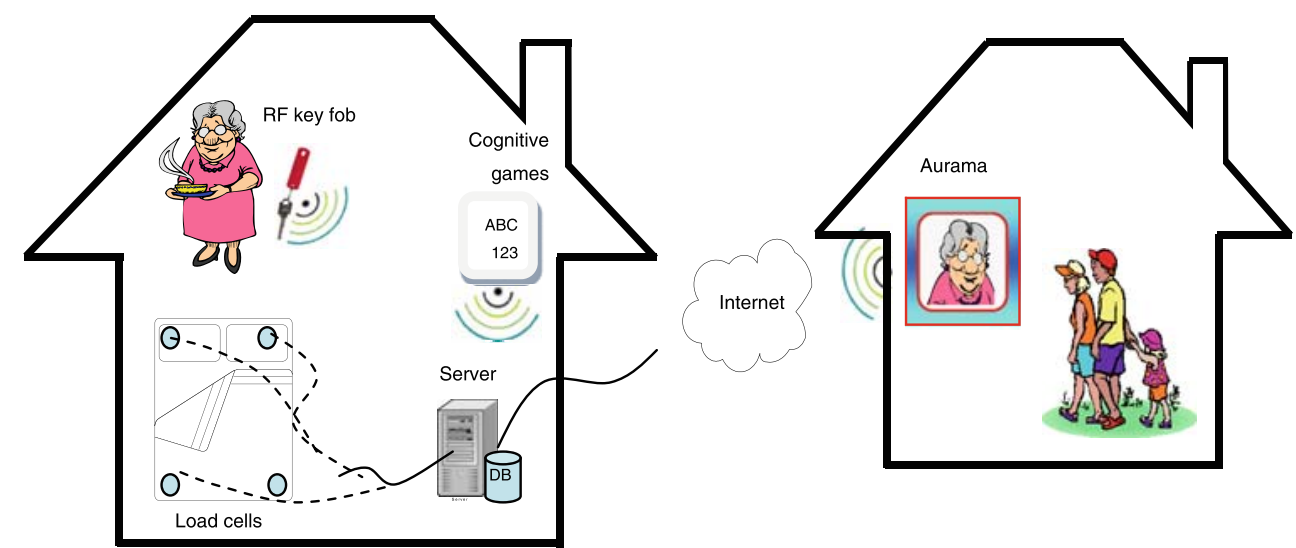




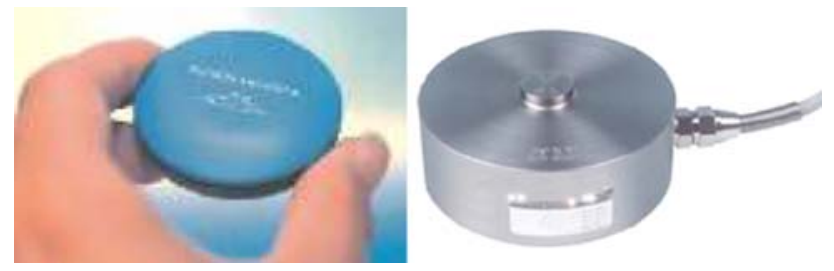

Fig. 4 The RF transmitter placed in a portable object like a key fob to detect presence (left) and the load sensor used under each leg of the bed to capture weight and sleep patterns (right)

By using load cells, we can unobtrusively monitor elder's weight without the need for the elder to stand on a weight scale every day. The same load sensors are used to monitor the number of times the elder gets up during the night, the amount of sleep, the time the elder goes to bed and the time the elder wakes up, to assess any irregularities. This information is sufficient to get a good indication of the quality of their sleep. By using load cells, we are able to monitor both the weight of elderly people and their sleep pattern with one set of sensors. The data collected during the night is assessed the day after to identify potential alert conditions based on predefined thresholds. These thresholds are dependent on the elder's lifestyle and are defined before using the system by asking the elderly about their usual lifestyle patterns. Alternatively, the system could run for several days in the beginning 'to learn' the patterns.

Cognitive ability is assessed with computer games that address different cognitive skills. The design and evaluation of these games is discussed extensively in (Cognitive Labs: http://cognitivelabs.com/). These games were deployed on a tablet PC given to the elderly. They would not only play the cognitive games on the PC, but also view their own wellbeing information on it. We chose to provide games that would assess the following skills (as explained in Gamberini et al. 2006; Zwartkruis-Pelgrim and de Ruyter 2008): mental rotation, memory, vigilance, selective attention and discrimination. Each skill is assessed with one game. Figure 5 shows an example of one of the questions in the mental rotation game, which presents users with threedimensional blocks and asks them to mentally rotate these blocks (Shepard and Metzler 1971). Each time the elder plays one of the cognitive games, they are asked to answer a set of five questions, which is considered to be one test. For
(A)

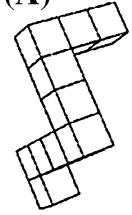

each test, a score is calculated according to the average number of correct answers and the average reaction time. For some games, the reaction time is more important than in others, therefore, a value between 0 and 1 is used to weigh the importance of reaction time for each game. Thus, for each cognitive skill an index score is calculated.

\subsection{Aurama: an aware picture frame}

Aurama includes a digital photo frame presenting a slideshow of personal pictures. The prototype uses a 7-inch tablet PC with a touch screen (see Fig. 1). Around it, a colored light 'aura' presents at-a-glance information regarding the elder's well-being. It is equipped with a stateof-the-art light guide made of transparent material making it look like a typical digital photo frame, with the addition of emitting colored light auras. If the elder is at home, a blue aura appears on the child's Aurama, which disappears if the elder leaves. To provide awareness of changes in well-being, a yellow or orange aura is formed, depending on the severity of the change.

When users interact with Aurama by touching the screen, they access an iconic menu screen shown in Fig. 6 with the choice to access sleep patterns, cognitive ability, weight patterns and an overview of well-being. The charts of Figs. 7, 8, 9, 10 show the detailed information collected about the elder that the user can browse through. Most charts show a long-term overview of a parameter relating to well-being. For example, sleep patterns (see Fig. 7) are presented as colored bars presenting the amount of sleep for each day for the previous 2 weeks. The color indicates whether it was a normal night of sleep or not, and two default severity levels are used: yellow and orange.

Trends in weight (see Fig. 8) show a daily weight trend compared to a running average. Thus, the time it takes for the weight to change is taken into account.

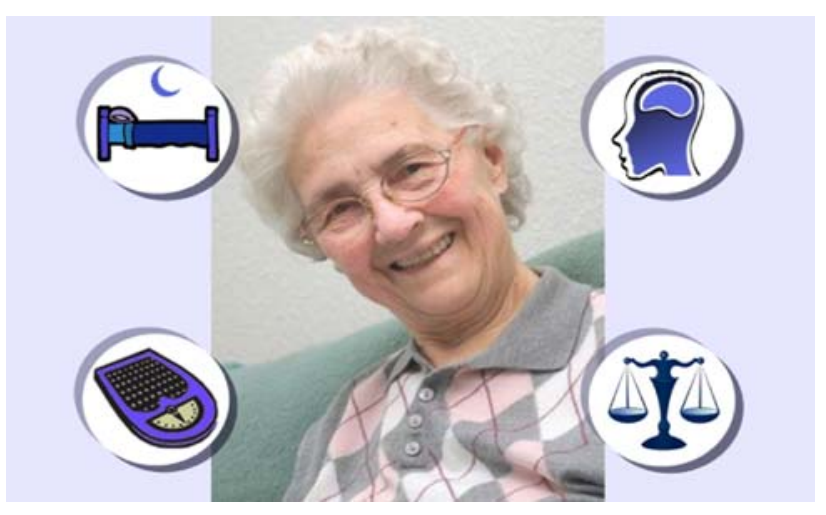

Fig. 6 The user interface for the menu screen: sleep patterns (top left), cognitive ability (top right), weight patterns (bottom left) and an overview of well-being (bottom right)
Fig. 5 Rotated blocks which are identical (left) and rotated blocks which are different (right)
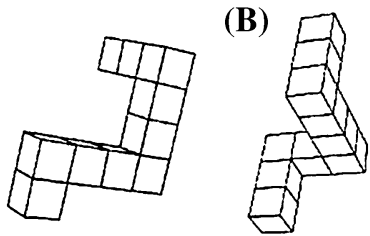

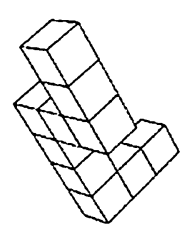


Fig. 7 The user interface for presenting sleep patterns

Fig. 8 The user interface for presenting weight trends

Fig. 9 The user interface for presenting trends in cognitive ability
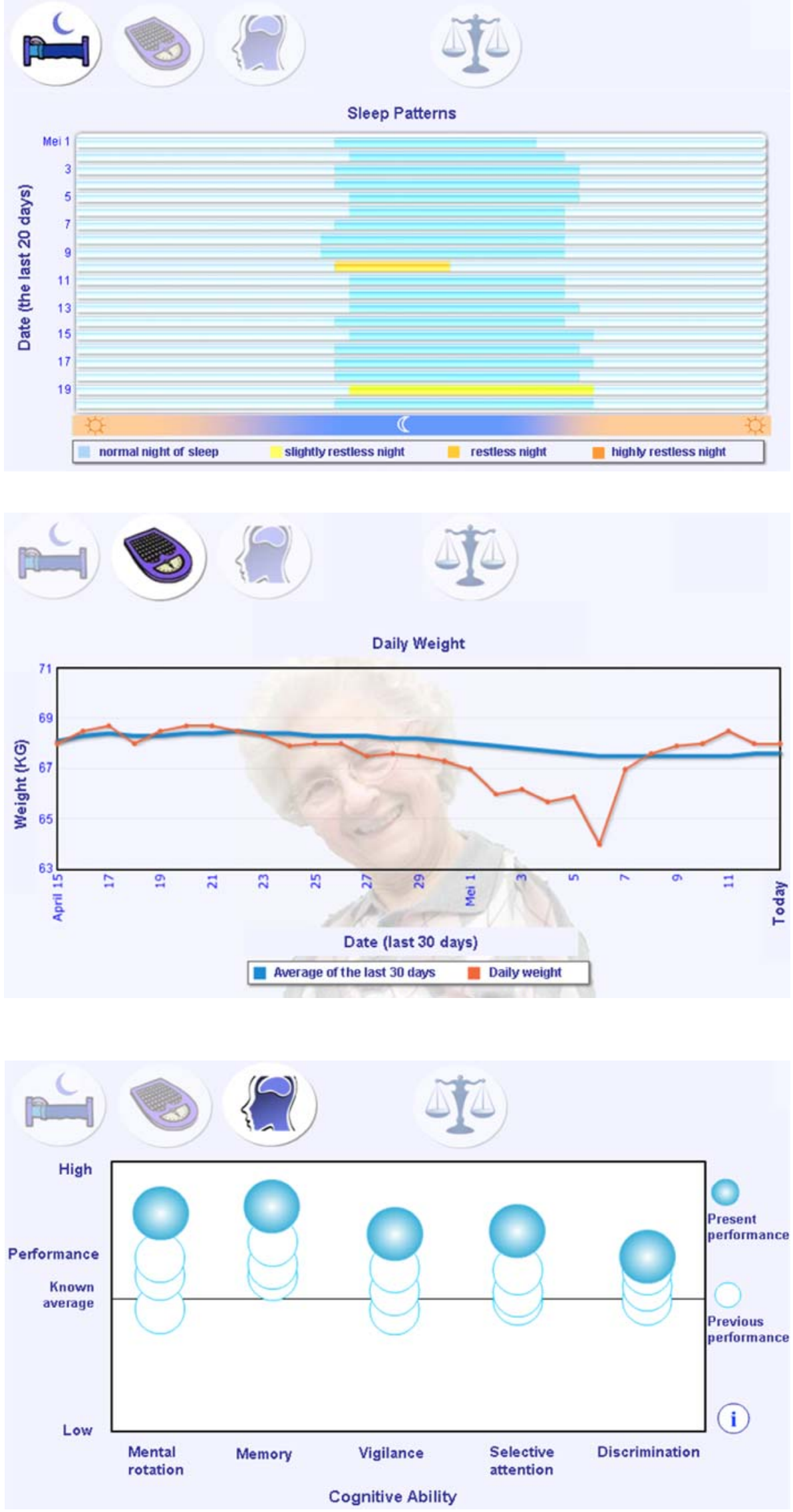


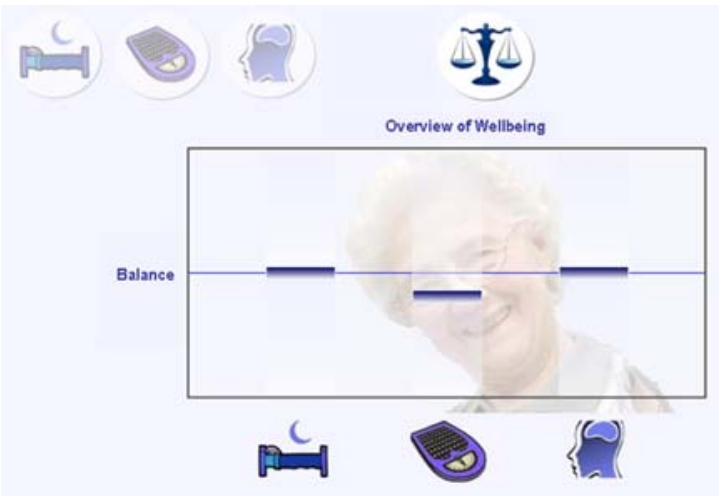

Fig. 10 The user interface of an overview to see if any parameter is 'out of balance'

For cognitive ability (see Fig. 9), different cognitive skills are shown in the form of blue and gray bubbles on the chart for the current and previous performances, respectively. Each bubble represents the average index score for five trials. Improvement or decline is observed by the position of the current performance with respect to previous performances.

Lastly, an overview of well-being (see Fig. 10) shows if any of the parameters is 'out of balance' with a bar being above or below a reference line. For sleep and weight being significantly above or below, the line could signify a health issue to be concerned about. For cognitive ability, the bar would only shift downwards if scores on the games start declining.

\section{Experience trials in a home lab setting}

A first user test ( $N=6$, average age: 46$)$ took place at the Philips Research ExperienceLab (http://www.research. philips.com/focused/experiencelab.html), a laboratory simulation of a home environment. The participants were peripherally involved caregivers. Two scenarios were evaluated using colored auras for abnormalities in weight and sleep trends.

The laboratory test showed a general appreciation of the application, which participants found easy to use. All participants were able to interpret the colored auras. When asked how they would react to information about the elder provided through the system, five of the six mentioned that they would call their parents to see how they are doing and one would wait and see if things go back to normal before giving a call. They indicated wanting more detailed information at times of abnormalities. All charts were well understood by the participants and the overview screen was preferred highly as a 'diagnosis' screen where it would be easy to spot what aspects of well-being have changed.
Users appreciated that the system would help them access well-being information more easily than through phone calls and visits. They expected they would gain 'peace of mind' and were positive that it would get them more involved in their parents' well-being. For privacy reasons, users wanted the option to choose which colors were used for certain abnormalities so that visitors would not be able to interpret them. Further, they expressed interest in getting information about the mood and emotional feelings of their parent, which they find difficult to assess with phone calls and visits.

Participants commented that knowing continuously when their parent is in or out may be distracting. They do not mind the display of presence information as long as they can disable it. They would feel obliged to respond when a colored aura would indicate an abnormality and in most cases they feel their parents would expect them to respond. However, they do not feel the system would create a burden on them and they reported that they would not mind feeling obliged to call in such situations. Several participants discussed that knowing about such changes in well-being would trigger more phone calls that could lead to more frequent visits. These results were found to be encouraging, but, given that the exposure to the system was brief, the preferences and opinions reported are rather hypothetical to the participants. For this reason, it was necessary to corroborate these findings with field trials.

\section{Field trials}

Two field trials were conducted to: (1) let users experience the system under realistic conditions and to evaluate its added value as a communication medium; (2) evaluate awareness and connectedness between parent and child and (3) evaluate privacy issues and technology acceptance. Each trial involved an elderly parent living alone, at a distance from a child who is peripherally involved in the care of the elder. The participants used the system for 2 weeks.

Questionnaire measurements were taken before installing the system, after using the system for 2 weeks, and 2 weeks after the system was removed. The participants were interviewed and filled in questionnaires to evaluate the ambient display such as the one used with the InfoCanvas (Stasko et al. 2004) and the affective benefits in communication (ABC) (Baren et al. 2003). These questionnaires provide an operational account of several positive and negative consequences regarding the use of awareness systems. It includes scales for the following affective benefits: awareness, connectedness, thinking of each other, usefulness, esthetics, novelty and fun, and the following costs: privacy, obligations, expectations, 
distraction. Each scale has four items, which users rate using a seven-point Likert scale ('strongly disagree' to 'strongly agree'). Some examples of such items were statements like: 'with the system I think about my mother more often than without the system', 'the system keeps me informed about my mother's well-being', 'with this system I feel more obliged to contact my mother than without the system' or 'with this system I feel a burden on me'. Some topics of informal discussion were about the effect on contacts between parent and child, privacy, bonding, and the use and value of the system. Furthermore, a web statistics tool was installed on each client to determine the use patterns.

\subsection{First field trial}

The first trial involved an 85-year-old woman living alone, $25 \mathrm{~km}$ from her daughter who lives with her husband and is peripherally involved in the care of her mother. Figure 11 shows the field trial set-up. The daughter interacted with Aurama, visiting each chart once a day on average to see how her mother was doing. Figure 12 shows the usage patterns of the awareness system for the elder and the child. She appreciated the presence information indicated with the colored auras very much and did not experience it as distracting. Every day, as she came back from work or before calling, she would check whether her mother was at home. She claimed that she did not need to know how many times her mother went out, but just to be aware of her presence was convenient and a nice experience. She claimed that presence information alone gave her a connected feeling with her mother.

During this trial, the elder did not sleep well during four nights. The first two were due to a death in the family, which caused her to get up several times. The other two nights were due to regular visits to the bathroom, one of those being due to stomach problems. Each time, a yellow aura was shown on her daughter's Aurama. The first two times, the daughter knew about the loss in the family and seeing that her mother did not sleep well, triggered her to call. For the other times, she did not call right away; instead she waited until her next routine call to inquire. She
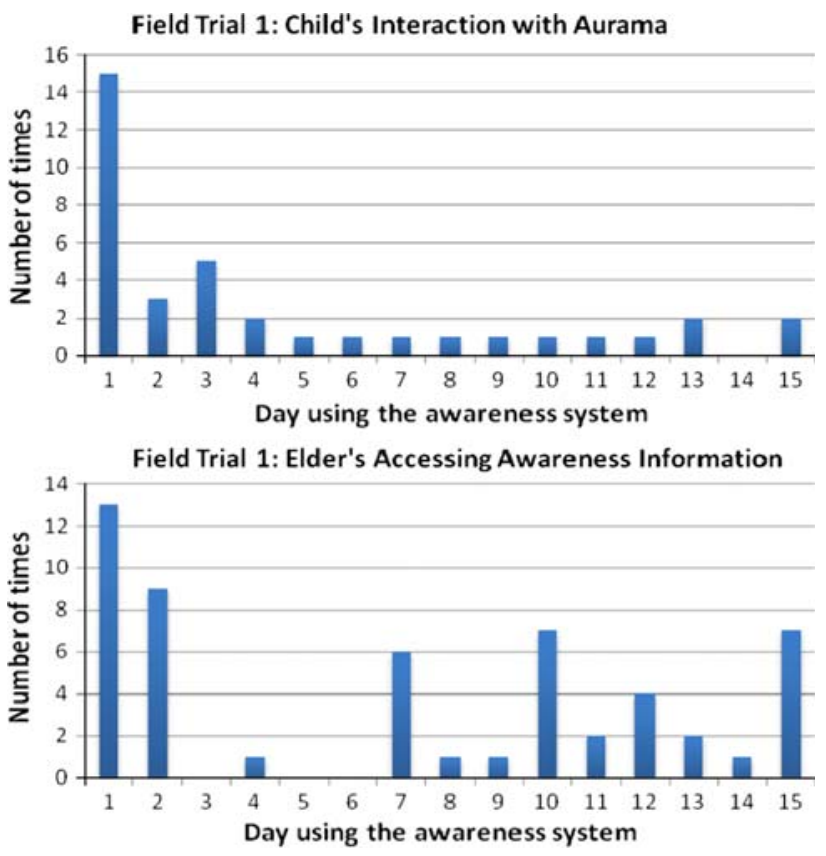

Fig. 12 Usage patterns of the first field trial for the child (top) and the elderly (bottom)

did not consider the system to be a burden nor felt that Aurama invaded privacy since her mother accepted to share the information. She wants her mother to have full control of what is shared.

The daughter was more interested in sleep and weight trends, and not in cognitive ability because she knew her mother was doing well mentally. It should be noted that information on cognitive ability cannot be appreciated very well given the short duration of this study. It is useful when in the period of use it reveals a trend that is not clearly perceived directly by the users. The daughter valued the charts as they provided a quick overview. If necessary, she would call to find out more details: "[...] not knowing the number of hours slept and exact times is ideal. It's good to have just an indication. If you want to know more, you should call. There should be more interaction between parent and child. It should trigger more contacts and not replace the contacts. If you call more, it could trigger you to visit more". She wanted additional information about
Fig. 11 Field trial 1 set-up: (left) the elder playing cognitive games on her photo frame, (center) the load cells under her bed and (right) Aurama in her daughter's living room indicating with a blue aura the presence of the elder
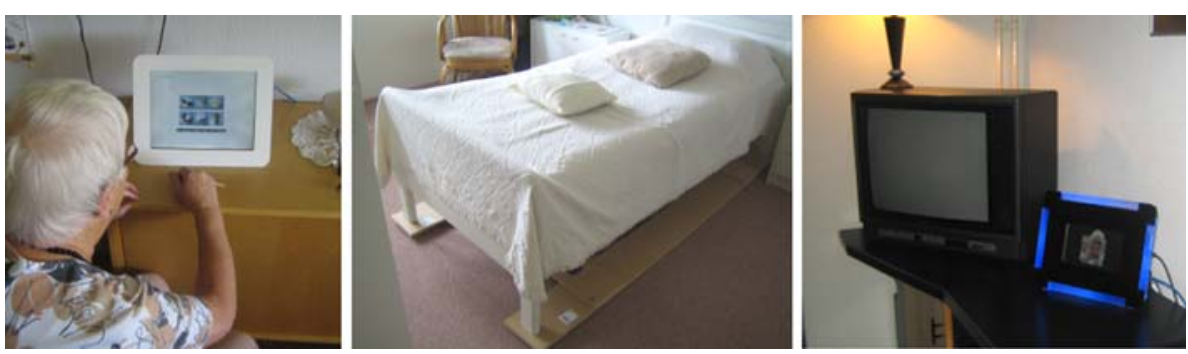
her mother's diabetes, eating patterns and medication. Furthermore, the son-in-law wanted the system for his mother because she is older, in a frailer condition, and lives alone in her own home.

The elder liked that her daughter called more since she likes it when her children show they care for her. However, she found the system to have more benefits for her daughter than for herself. Although she thought the system should be targeted at frailer elders, she was happy that her daughter knew about her well-being so she would not worry. She did not want alerts for trivial events, e.g., a bad night of sleep, but rather for repeated or more serious events. She found it amusing when her daughter asked her about a bad night of sleep. She appreciated the fact that not too many details were given, like the number of toilet visits.

Morris et al. (2003) suggest that elderly should interact through interfaces they are familiar with like ones embedded in everyday objects (e.g., a photo frame). However, our research reveals that this is not enough. Adopting new technology is harder when it replaces another technology they are familiar with. For example, although the elder was asked to play the cognitive games on her photo frame, she still preferred to play her own paper-based games.

\subsection{Second field trial}

The participant in this trial was an 81-year-old man living alone about $30 \mathrm{~km}$ from his son (married with three children). The elder was always concerned about putting a burden on his children. He called them only when necessary rather than making routine calls to keep in touch. During the trial, he preferred to have the presence tag in his wallet and by misfortune he forgot his wallet once and was out all day. His son called him several times since there was a presence aura on Aurama indicating he was present at home. Not having his calls answered, he panicked and drove to his father's place to check on him. He experienced a negative effect for worrying unnecessarily by finding his father non-responsive while being informed about his father's presence at home.

The elder had no problems with sharing information with his son. He found the system useful but was nervous about forgetting his wallet again. He experienced three abnormal nights of sleep. Every time, a yellow aura was shown on Aurama and the son called. He preferred to have more details on sleep to see what was not normal. Contrary to the first trial, he would like the system to give him all the details rather than him needing to inquire about them. The grandchildren (under 10) also reacted to the alerts by calling it to the attention of their parents.

The caregiver was clear about wanting to be informed only about serious situations. He appreciated the idea of having only the overview screen to check if everything is fine, but have access to all details if something is wrong. He found it annoying to be informed whenever his father had one bad night of sleep: "inform me only if he has not slept well for 4 or 5 nights", showing a preference for a macroscopic view of the situation of the parent. He preferred to access this type of information on a PC rather than a photo frame because he found the constant aura on Aurama distracting, and thus he felt 'forced' to look into the charts several times a day. Figure 13 shows the usage $\operatorname{logs}$ for the second field trial. It is interesting to note that the elder was quite active since he was encouraged by his son to play the cognitive games to assess his cognitive ability and how that changes over time.

\section{Discussion}

Aurama represents a refinement over earlier works in using a picture frame based display of awareness information (Consolvo et al. 2004; Mynatt et al. 2001; Rowan and Mynatt 2005). From a design perspective, it pays more attention to esthetics and in insuring that it offers an unobtrusive, peripheral way of indicating presence and trends in well-being of a single elderly person. Test users appreciated the design esthetics of the display and the colored auras to indicate status information of a close relative.

More importantly, the design and evaluation of Aurama were aimed at identifying the nature of the information that is required to provide support in the scenario of a single elder and a remote, care giving child. The analysis of the
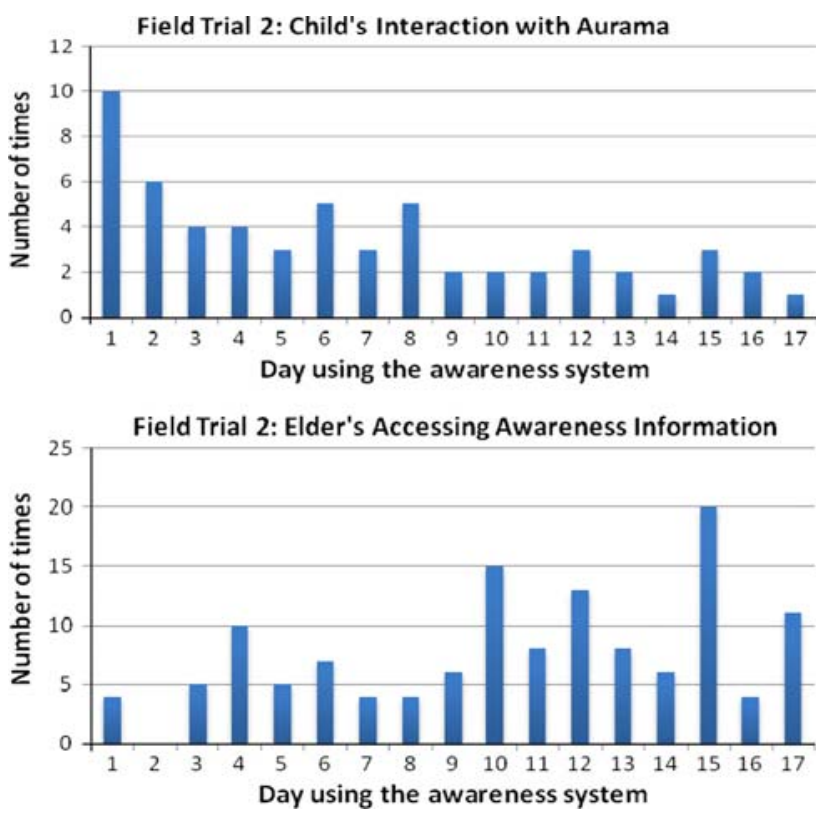

Fig. 13 Usage patterns of second field trial for the child (top) and the elderly (bottom) 
user needs at the beginning of the project, contrasts earlier approaches, which are based on providing a detailed account of activity levels of the elderly (Mynatt et al. 2001; Rowan and Mynatt 2005) or of the activities themselves (Metaxas et al. 2007). Specifically, in our study, the children indicated that they want to know about changes in well-being that are difficult to assess during visits and phone calls, i.e., long-term trends, moods and, in accordance with earlier findings (Brown et al. 2007; Khan and Markopoulos 2009), the general reassurance that things are going as anticipated. Given the expressed preference of this group, Aurama was designed to display the long-term evolution of important, objective, and sometimes very personal parameters relating to the well-being of the elder. These parameters helped to alert caregivers to unusual situations also.

Contrary to earlier work (Metaxas et al. 2007), we found that the use of narratives or 'stories' with detailed information (what, when, where) was generally disliked by adult children and was seen as intrusive. Users appreciate that Aurama does not give very detailed information and emphasize that it provides them with just enough high level information.

Although the charts show long-term trends of well-being parameters, the participants used the system for 2 weeks only, which is not enough time to accumulate enough data to identify what constitutes an abnormal change. Therefore, to assess how the children would react to the colored auras indicating an abnormal change, the alerts in the trial had to be triggered by one-time events (e.g., one bad night of sleep). However, participants clearly indicated that based on their experience the alerts should be given only for longterm or persistent abnormalities.

Privacy is a major concern in designing awareness systems. Our studies show that the children do want to know about their parent's well-being but often fear that they are intruding, even when their parents do not mind sharing the information.

The system focused on negative changes in well-being. Participants in the user studies reported a major interest in being aware of the positive aspects or the improvements in well-being as well. This could indeed foster further peace of mind. There was interest shown to augment the photo frame further to exchange messages or to be able to see each other and assess well-being via a video link.

The field trials also demonstrated that the participants were very interested in self-awareness, which is consistent with the findings reported by Morris et al. (2003). Elderly are interested in very detailed information about their wellbeing for themselves, but want to share only just enough with their children to provide peace of mind to them.

As noted in the second field trial, the shown awareness information needs to be reliable. The benefits of an awareness system that aims for peace of mind can be canceled out completely or even reversed, if erroneous data is given. It is therefore crucial not to depend on one approach to context sensing but to combine different technologies to increase reliability and robustness of the system. This is consistent with the experiences reported for the DFP (Rowan and Mynatt 2005) and the Diarist (Metaxas et al. 2007). From a technical perspective, reliability and robustness are essential; more interestingly, interaction designers need to find solutions to address these problems by providing graceful degradation when things go wrong and useful feedback regarding the operation of the system, see for example (Metaxas et al. 2007) where the notion of seamful design was explored.

Regarding the use of sensors and the algorithms, we encountered some situations that we did not anticipate. For example, it was noticed that the algorithms for sleep detection were too simple. As soon as a person was on the bed, whether sitting, lying or reading, it was recorded as going to sleep and if and when the person would subsequently get off the bed it would be recorded as a wake-up event. So even though the elder may have sat down on the bed for only 5-s just before going to sleep, the system would record a get-up event. The elderly in the second field trial appeared erroneously to have quite an irregular sleeping pattern due to frequent sitting down and getting up before actually going to sleep. Also, he always reads a lot in bed before falling asleep, the system recorded this as him being asleep already. A proper detection algorithm is needed that distinguishes between sitting on the bed, lying on the bed, reading on the bed, sleeping, leaning on the bed and so forth. It is anticipated that this can be addressed by analyzing the data coming from each load cell individually and assessing if the person is moving or is relatively motionless.

Furthermore, although the load cells are calibrated once a day, the indicated weight is never $0 \mathrm{~kg}$ just before the elderly goes to bed. It is usually a few kilos less, so it could be that bed sheets are removed. The lack of accuracy in this case can be resolved by calculating the difference in measured weight just before and just after getting up from bed, rather than taking the absolute measured value during the night and/or by taking into account events like taking blankets and other objects from and to the bed before and after going to bed.

With presence detection, it happened a couple of times that the sensor was 'thresholding', meaning that the sensor was located at the limit of the signal detection zone and would register in and out events several times in short succession, causing a blue aura to appear on the child's photo frame and disappear within a short period of time.

The settings and thresholds for the normal number of wake-ups during the night was based on a questionnaire and entered into the system manually. It would have been 
better if an automated system with a learning feature could have been implemented.

The above problems are typical for the development and deployment of sensor technology and illustrate some of the challenges that need to be overcome during field testing and need to be accounted for in the system and interaction design.

A database management system has proven to be an efficient tool to manage all the data of the system and to provide the interface between different components of the system. Using a database for sensor events and derived conclusions has proven to be effective to support long-term trend analysis of the data about the elderly.

\section{Conclusions and future work}

The presented research explored the potential of ambient intelligence to support awareness of context information of elderly living alone. Aurama targets adults who are peripherally involved in the care of their aging parents. We have provided evidence regarding the value of awareness systems for this target group, in line with the earlier works mentioned in the introduction (Consolvo et al. 2004; Mynatt et al. 2001; Metaxas et al. 2007; Morris et al. 2003). Our system provides a rich and personal set of information, in a way that makes it easy for the children to get an insight in the long-term trends in the well-being of their elderly parents. Aurama also provides presence information and offers the elders the ability to determine how their wellbeing information is presented.

Contrary to prior work, we have found that people in the targeted user group, and in the social context we looked at, prefer to get long-term trend information regarding the well-being of their parent rather than low-level information regarding whereabouts, activities, or social interactions. This result suggests reorienting related research toward sensing and visualizing long-term data. It also indicates the importance of long-term field trials.

Our work emphasizes the esthetic aspects of awareness information displays. The innovative and esthetic design of Aurama features colored light auras around a photo frame that allow users to get an overview of the state and wellbeing of their parent at-a-glance without demanding to be the focus of their attention. Especially, the combination of the long-term trend data and our new way of presenting data with colored light auras were appreciated by participants in the field trials.

Our implementation focused on presenting meaningful and personal awareness information, in particular, sleeping patterns, weight variations and cognitive decline. From the evaluation, we found that sharing this information is valued for achieving connectedness and peace of mind, at the same time, users want mechanisms to control the associated information flow. Further research is needed to develop and evaluate such mechanisms and to establish a more uniform acceptance regarding the methods used to capture the information.

Although the elders are able to inspect the information shown about them, Aurama is in essence a one-way information channel. Future versions of the system could explore which information the elder may want to know regarding their children. There is an opportunity for making the system symmetric and support social translucence (Erickson and Kellogg 2000), that is, the notion of being 'monitored' is reduced or even removed.

In literature, there is not much reported about the patterns of use of awareness systems for our target user groups. Earlier work (Consolvo et al. 2004; Mynatt et al. 2001; Rowan and Mynatt 2005; Metaxas et al. 2007) reported promising initial findings, but the number and duration of trials are still limited. It is of interest to investigate what users infer from the awareness information, in particular from long-term trends in parameters related to well-being, and to explore if children and elderly continue to use the system after an extended period of time. Our studies have shown that children are interested in being aware of the mood and state of mind of their elderly parent, but it is still unclear what the emotional benefits of these would be. Furthermore, there is a profound interest in awareness of positive aspects of well-being and further research is needed to adapt the Aurama awareness system to accommodate these needs. Furthermore, elderly want to use the system as a means of exchanging asynchronous messages or synchronous video chats with their loved ones, but the potential downside of these (e.g., lower number of phone calls and/or visits) should be further explored.

Acknowledgments The authors would like to thank Simon Abernethy for his work on implementing the awareness system and the participants from the various user studies. The TU/e research is supported by the FET IST program, project ASTRA, IST 29266.

Open Access This article is distributed under the terms of the Creative Commons Attribution Noncommercial License which permits any noncommercial use, distribution, and reproduction in any medium, provided the original author(s) and source are credited.

\section{References}

Aarts E, Marzano S (eds) (2003) The new everyday: visions of ambient intelligence. 010 Publishing, Rotterdam

Aarts E, Harwig H, Schuurmans M (2001) Ambient Intelligence. In: Denning J (ed) The invisible future. McGraw-Hill, New York, pp 235-250

Adami A, Hayes T, Pavel M (2003) Unobtrusive monitoring of sleep patterns. In: Proceedings of EMBS, pp 1360-1363 
Baren J, IJsselsteijn W, Romero N, Markopoulos P, Ruyter B (2003) Affective benefits in communication: the development and fieldtesting of a new questionnaire measure. In: Proceedings of PRESENCE

Brown BAT, Taylor AS, Izadi S, Sellen A, Kaye J, Eardley R (2007) Locating family values: a field trial of the whereabouts clock. In: Krumm J, Abowd GD, Seneviratne A, Strang T (eds) Ubicomp, volume 4717 of Lecture Notes in Computer Science. Springer, Berlin, pp 354-371

Chalmers M, Galani A (2004) Seamful interweaving: heterogeneity in the theory and design of interactive systems. In: Proceedings of DIS, pp 243-252

Consolvo S, Roessler P, Shelton B (2004) The CareNet display: lessons learned from an in home evaluation of an ambient display. In: Proceedings of Ubicomp, 2004

Erickson T, Kellogg W (2000) Social translucence: an approach to designing systems that support social processes. ACM Transactions on Computer-Human Interaction 7:59-83

Gamberini L, Alcaniz M, Barresi G, Fabregat M, Ibanez F, Prontu L (2006) Cognition, technology and games for the elderly: an introduction to ELDERGAMES Project. PsychNology Journal 4:285-308

Kelley JF (1984) An iterative design methodology for user-friendly natural language office information applications. ACM Transactions on Office Information Systems 2(1):26-41

Khan V, Markopoulos P (2009) Busy families' awareness needs. Int J Hum Comput Stud 67(2):139-153
Metaxas G, Metin B, Schneider J, Markopoulos P, de Ruyter B (2007) Daily activities diarist: supporting aging in place with semantically enriched narratives. In: Proceedings of INTERACT

Morris ME (2005) Social networks as health feedback displays. IEEE Int Comput 9(5):29-37. doi:10.1109/MIC.2005.109

Morris M, Lundell J, Dishman E, Needham B (2003) New perspectives on ubiquitous computing from ethnographic study of elders with cognitive decline. In: Proceedings of Ubicomp, pp 227-242

Mynatt E, Rowan J, Craighill S, Jacobs A (2001) Digital family portraits: supporting peace of mind for extended family members. In: Proceedings of CHI, pp 333-340

Nakamura EF, Loureiro AAF, Frery AC (2007) Information fusion for wireless sensor networks: methods, models, and classifications. ACM Comput Surv 39(3):9

Rowan J, Mynatt E (2005) Digital family portrait field trial: support for aging in place. In: Proceedings of CHI, pp 521-530

Shepard RN, Metzler J (1971) Mental Rotation of three-dimensional objects. Science 171:701-703

Stasko J, Miller T, Pousman Z (2004) Personalized peripheral information awareness through information Art. In: Proceedings of Ubicomp, pp 18-35

Zwartkruis-Pelgrim E, de Ruyter B (2008) Developing and Adaptive Memory Game for Seniors. In: Markopoulos P et al (eds) Fun and Games 2008. LNCS, Springer 